Historic, Archive Document

Do not assume content reflects current scientific knowledge, policies, or practices. 
All goods put f. o. b. cars Orange, Conn. or f. o. b. cars or steamer, New York City.

Not bound by these prices for any definite time or quantity and subject to advance without notice; $10 \mathrm{lbs}$. and over at $100 \mathrm{lb}$. rate.

\section{TERMS OF CREDIT}

Bills are due two months from date of invoice, or subject to a discount of one and one-half per cent. for cash within ten days of date of invoice.

An additional charge of $5 \mathrm{c}$ per $1 \mathrm{~b}$. will be made on orders for less than $10 \mathrm{lbs}$. of a variety.

Unknown correspondents desiring credit are requested to give satisfactory reference.

Messrs. S. D. Woodruff \& Sons give no warranty, expressed or implied, as to description, quality, productiveness, or any other matter of any seeds, plants, bulbs, etc. it sells, and will not be responsible for the crop.

\section{S. D. WOODRUFF \& SONS}

\section{Orange, Conn.}

Telephone Orange 17-12

New Haven, Colony 2200-2

\section{Washington St., New York City}

New York Tel., Barclay 0690

We are members of and use Telegraph Code of American Seed Trade Association. 


\section{ASPARAGUS}

Palmetto

Giant Argenteuil

Washington Blight Resistant

Mary Washington Blight Resistant

Mary Washington is an Improved Washington

\section{ASPARAGUS ROOTS}

Strong 2-year roots of all above varieties $\$ 1.50$ per $100, \$ 10.00$ per 1,000 .

Washington and Mary Washington 2-year roots, $\$ 2.00$ per $100, \$ 12.00$ per 1,000 .

\section{BEANS-DWARF BUSH OR SNAP-GREEN PODDED}

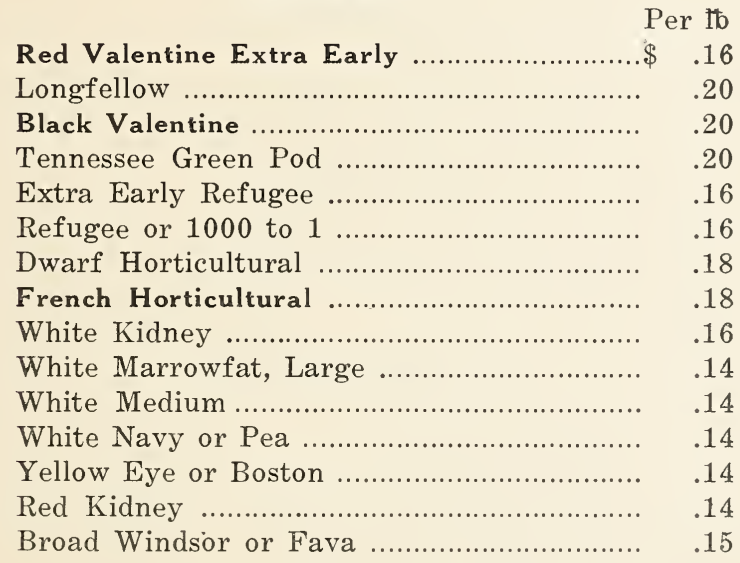

\section{BEANS-STRINGLESS GREEN POD VARIETIES}

Bountiful \$ $\quad .25$

Burpee Stringless Green Pod ..................... .25

Giant Stringless Green Pod ....................... .25

Refugee Rogers Stringless ........................ Sold out

Full Measure XX ..................................... $\quad .30$

Lowe's Champion ...................................... .25

\section{BEANS-DWARF WAXSORTS}

Stringless Refugee Wax ......................

Davis White Kidney Wax ........................ $\quad .20$

Currie's Rust Proof Wax .......................... .22

Wardwell's Kidney Wax .......................... $\quad .18$

Improved Golden Wax ............................. $\quad .18$

Golden-Eyed Wax ................................. 18

Pencil Pod Black Wax .............................. $\quad .18$

Hodson Wax ............................................ $\quad .16$

Round Pod Kidney Brittle Wax .................. $\quad .22$

Sure Crop Wax ..................................... .22

Webber or Crackerjack Wax ..................... $\quad .22$

Burpee's New Kidney Wax ....................... $\quad .22$

Unrivaled Wax .......................................... . 22 


\section{BUSH LIMAS}

Burpee's Bush Limas

Per ib

Burpee's Improved Bush Limas .....

\$ .20

Dreer's Bush Limas

.20

Fordhook Bush Limas

.20

Henderson's Bush Limas

\section{POLE LIMAS}

Carpenteria Pole Limas

Per it

Challenger Pole Limas

Dreer's Improved Pole Limas .................... $\quad .20$

Early Jersey Pole Limas ............................. $\quad .20$

Early Leviathan Pole Limas ..................... $\quad .20$

Extra Large White Pole Limas .................. $\quad .20$

Ford's Mammoth Pole Limas ..................... $\quad .20$

Giant Podded Pole Limas ........................... $\quad .20$

King of the Garden Pole Limas ................ $\quad .20$

Large White Pole Limas ........................... $\quad .20$

New Ideal Pole Limas ................................ $\quad .20$

Seibert's Early Pole Limas ......................... $\quad .20$

Small Sieva Pole Limas ............................ $\quad .20$

Ideal Pole Lima ........................................ $\quad .20$

\section{POLE BEANS}

Dutch Case Knife

Kentucky Wonder

Horticultural Mamoth Pod ......................... $\quad .18$

Lazy Wife ............................................... $\quad .18$

Golden Cluster Wax ................................. 18

Cutshort Speckled ....................................... .18

Kentucky Wonder Wax ............................ $\quad .20$

Kentucky Wonder White Seed .................... $\quad .20$

Scarlet Runners ................................... $\quad .20$

White Dutch Runners .............................. $\quad .20$

Striped Creaseback ................................... $\quad .20$

White Creaseback ................................... $\quad .20$

\section{BEETS}

Early Wonder

Crosby's Egyptian .................................. $\quad .40$

Crimson Globe ....................................... $\quad .40$

Early Dark Red Flat Egyptian .................. $\quad .40$

Eclipse ................................................. .40

Detroit Dark Red .................................... . .40

Early Blood Turnip .................................. $\quad .40$

Edmund's Blood Turnip ............................ $\quad .40$

Half Long Blood ....................................... $\quad .40$

Long Smooth Blood .................................. $\quad .40$

Bassano ................................................. $\quad .40$

Swiss Chard (Green) ............................. $\quad .40$

Swiss Chard (Lucullus) .......................... $\quad .40$ 


\section{SUGAR BEETS AND MANGELS}

10 ib or over, 5 cents a ib less than printed price.

Per it

Mammoth Long Red ................................. $\quad .35$

Golden Tankard ..................................... . 35

Yellow Globe ............................................ . 35

Giant Half Sugar Rose ............................. $\quad .35$

Klein Wanzleben Sugar Beet .................. $\quad .35$

\section{BRUSSELS SPROUTS}

Per ito

Improved Half Dwarf

$\$ 1.75$

Long Island Imp. Dwarf

2.00

\section{BROCCOLI}

Per $1 \mathrm{~b}$

White Cape

$\$ 2.00$

Large Green Calabrese

7.00

\section{CABBAGE}

Per ib

Early Jersey Wakefield

$\$ 1.50$

Large or Charleston Wakefield

1.50

Copenhagen Market

2.00

Golden Acre, New

3.00

Early Winnigstadt (best imported)

1.50

All-Head Early

1.50

Early Summer

1.50

Succession

1.50

All Seasons

1.50

Sure Head

1.50

Steins Early Flat Dutch

1.50

Premium Flat Dutch

1.50

Perfection Drumhead Savoy

2.00

Danish Ball Head, Short Stem

2.00

Danish Ball Head, Long Stem

2.00

Mammoth Red Rock

2.00

Glory of Enkhuizen (Imported)

2.00

Chinese Wong Bok

2.50

\section{CARROTS}

Per ito

Coreless

$\$ \quad .70$

Chantenay Half Long Scarlet .................... $\quad .60$

Early Short Horn ...................................... $\quad .60$

St. Valery ............................................... $\quad .60$

Scarlet Nantes ...................................... $\quad .70$

Oxheart .................................................... $\quad .60$

Danver's Improved Half Long ................. $\quad .60$

Improved Long Orange ............................. $\quad .60$

Yellow Belgian ........................................ $\quad .60$

White Belgian .......................................... $\quad .60$

Long Danvers (new select) ..................... 1.00

Hutchinson (True) ................................. 2.00

New Bagley Danvers .............................. 1.00

See Market Gardener's List 


\section{CAULIFLOWER}

\begin{tabular}{lrr} 
& Per oz. & \multicolumn{1}{c}{ Per tb } \\
Snowball .............................\$ & 1.50 & $\$ 16.00$ \\
Extra Early Dwarf Erfurt .......\$ & 1.50 & 16.00 \\
Veitch's Autumn Giant ............. & .60 & 6.00 \\
Early Paris ............................ & .60 & 6.00 \\
Danish Giant ........................ & 1.50 & 16.00 \\
Dry Weather ........................ & 1.50 & 16.00
\end{tabular}

\section{CELERY}

Per itb

Wonderful

$\$ 9.00$

Golden Plume

9.00

Paris Golden Self-Blanching best French ....

5.00

Golden Self-Blanching, American

2.50

Giant Pascal Select

1.20

White Plume

1.50

Dwarf Golden Heart

1.00

Easy Blanching (Sanford Superb) ............ 1.50

Boston Market ....................................... 1.00

Winter Queen ......................................... 1.00

Woodruff's Beauty .................................. 1.20

Smallage or Cutting Celery ...................... 1.20

Old Seed for soup flavoring ...................... .25

Turnip Rooted Celeriac ............................. 1.50

\section{CHICORY}

Per 南

$\$ 1.00$

Whitloof, French Endive

1.25

\section{COLLARDS}

True Georgia

Per ib

.$\$ 50$

\section{SWEET CORN}

Sweet Corn is one of our great specialties; we can supply the very best stocks in quantity. Connecticut Sweet Corn leads the world for quality.

Per 100

Selected

Early Columbia

Ears $100 \mathrm{lb}$

Early Surprise

$5.00 \$ 15.00$

Large Early Adams

5.00

Extra Early Adams

Norfolk Market

Trucker's Favorite

Howling Mob

12.00

Early Mayflower

Early Champion

$\begin{array}{ll}5.00 & 12.00 \\ 5.00 & 11.00\end{array}$

Metropolitan

$5.00 \quad 11.00$

White Cob Cory

Long Island Beauty

$5.00 \quad 11.00$

$10.00 \quad 14.00$ 


\section{SWEET CORN-Continued}

\begin{tabular}{|c|c|}
\hline Early Minnesota & 10.00 \\
\hline Black Mexican ..... & \\
\hline Crosby's Early .... & \\
\hline Early Evergreen & 5.00 \\
\hline Kendall's Early Giant ...... & 5.00 \\
\hline Stowell's Evergreen ......... & 5.00 \\
\hline New White Evergreen ...... & 5.00 \\
\hline Narrow Grain Evergreen & \\
\hline ry Gentleman .................. & 5.00 \\
\hline 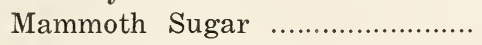 & 10.00 \\
\hline Sweet Fodder Corn & \\
\hline
\end{tabular}

\section{GOLDEN SEEDED SORTS}

Per 100

Selected

\begin{tabular}{|c|c|c|}
\hline & Ears & $100 \mathrm{16}$ \\
\hline nshine XX & 7.00 & $\$ 20.00$ \\
\hline oodruff's Extra Early & 10.00 & 20.00 \\
\hline e Burpee ............ & 10.00 & 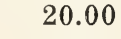 \\
\hline olden Rod XX & 7.00 & \\
\hline t Orange & 7.00 & • \\
\hline n Evergreen & 8.00 & 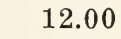 \\
\hline Giant & 7.00 & 2.00 \\
\hline les Early Yello & 7.00 & .0 \\
\hline ler & 5.0 & \\
\hline
\end{tabular}

\section{POP CORN}

White Rice

$\begin{array}{llll}\$ & .15 & \$ & .09\end{array}$

\section{FIELD ENSILAGE CORN}

Per bu.

Extra fine quality this year; strong vitality. Write for price on $50 \mathrm{bu}$. or over.

Early 8-Rowed Yellow Canada

Longfellow

Sanford White Flint

Improved Leaming 2.00

Early Mastodon 2.25

Pride of the North 2.25

Eureka Ensilage 2.75

Red Cob Ensilage 2.25

Reid's Yellow Dent 2.25

Cuban Giant 2.50

White Cap Dent 2.25

Sweepstake XX

3.00

Woodruff's Beauty Silo

3.00

CORN SALAD

Large Seeded

\section{CRESS}

Fine Curled or Peppergrass 


\section{CUCUMBERS}

Early Russian

Per $1 \mathrm{tb}$

Chicago Picklin

$\$ \quad .70$

Boston Pickling

.60

Davis Perfect

Early Cluster

Woodruff's Hybrid

Early Fortune

Early Green Prolific ................................. $\quad .60$

Early White Spine ................................. $\quad .60$

Extra Long White Spine ........................ .60

Arlington White Spine ............................ $\quad .60$

London Long Green ................................ $\quad .70$

Fordhook Famous ................................... $\quad .70$

\section{DANDELION}

Per ito

Improved Large Leaved

$\$ 7.00$

French Common

\section{EGG PLANT}

N. Y. Improved Spineless

Per itb

Black Beauty

$\$ 3.50$

Florida High Bush

3.50

Long Purple

3.50

3.50

\section{ENDIVE}

Per itb

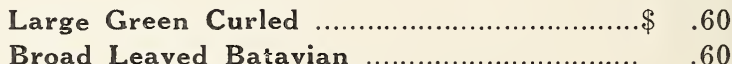

White Curled ........................................... $\quad .60$

\section{GOURDS}

Nest Egg

Per itb

$\$ 1.00$

Sugar Trough .......................................... 1.00

Dipper ................................................. 1.00

Hercules Club ........................................ 1.75

Mixed all sorts ................................... $\quad .90$

KALE

Per $\mathrm{ib}$

Dwarf Curled Scotch ................................ $\quad .45$
Tall Curled Scotch ............................... .50

Dwarf Siberian or Blue Kale ..................... $\quad .30$

\section{KOHL RABI}

\section{LEEK}




\section{LETTUCE}

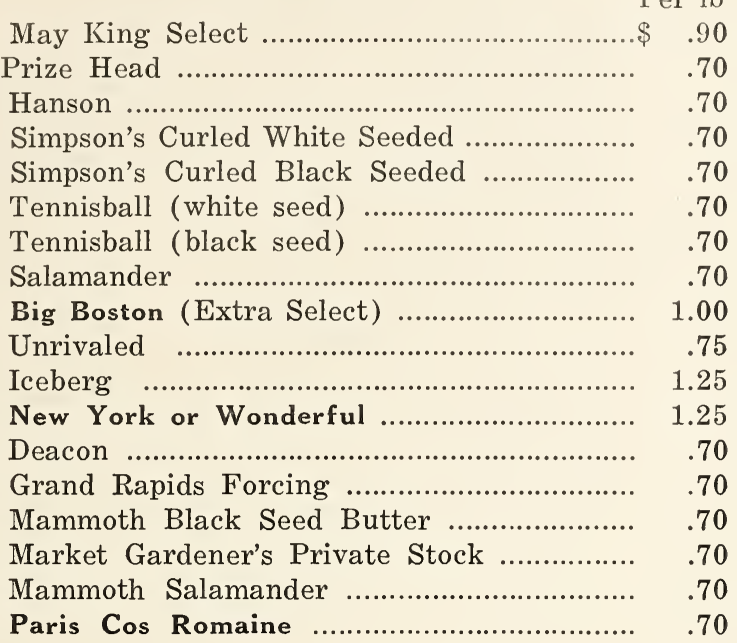

\section{MUSK MELON}

Paul Rose

Miller's Cream

.60

Osage Salmon Fleshed

.70

Emerald Gem

.70

Rocky Ford

.60

Long Island Beauty

.60

Hackensack

.70

Extra Early Hackensack

.70

Baltimore or Acme

.60

Bender's Surprise

Tip Top

Jenny Lind

.60

Burrell's Gem

The Grand

Banana

Fordhook

Pollock No. 25 .......................................... $\quad .70$

Hoodoo

Eden Gem

Honey Dew

\section{WATERMELON}

Hungarian Honey

Phinney's Early ......

Irish Grey

Sweet Heart

Peerless or I

Mammoth Ironclad

Mountain Sweet 


\section{S. D. WOODRUFF \& SONS}

\section{WATERMELON - Continued}

Cole's Early

Florida Favorite

Alabama Sweet

Tom Watson

Citron Red Seed

Fordhook Early

Dixie

Stone Mountain

\section{PARSLEY}

Moss Curled

Per $1 \mathrm{tb}$

Triple Curled

$\$ \quad .50$

Plain Leaved

Hamburg or Hermisher Turnip Rooted

\section{PEAS}

\section{Extra Early Round Varieties}

First and Best

Alaska

Pilot, Round Seeded Gradus

\section{PEAS}

\section{Extra Early Wrinkled}

Nott's Excelsior

American Wonder

Premium Gem

Blue Bantam

Gradus

Thomas Laxton

Little Marvel

Laxtonian

Sutton's Excelsior

Laxton's Progress

World's Record

Laxton's Superb

Pioneer

Hundredfold

Marchioness

\section{PEAS}

\section{Second Early and Main Crop}

Alderman

Prince Edward

14

Horsford's Market Garden ....................... $\quad .12$

Advancer Improved ................................ .12

Yorkshire Hero .......................................... .12

Champion of England ............................... $\quad .12$

Bliss Everbearing ..................................... $\quad .12$ 


\section{PEAS - Continued}

Carter's Daisy or Dwarf 'Telephone .......... $\quad .16$

Dwarf Sugar ............................................. 18

Mammoth Melting Sugar .......................... .20

White Marrowfat ..................................... 12

Canada Field ............................................ $\quad .06$

\section{PEPPER}

\begin{tabular}{|c|c|}
\hline & \\
\hline loodruff's Colossal & \\
\hline Neapolitan & \\
\hline et Mountain & \\
\hline King ............ & \\
\hline Nose or Bell & \\
\hline e Giant. & \\
\hline 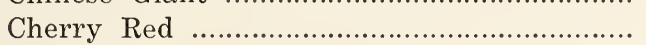 & \\
\hline ed Chili & \\
\hline ong Red Cay & 3. \\
\hline Giant & 3.0 \\
\hline ................ & 3. \\
\hline$\cdots$ & 4. \\
\hline nen & \\
\hline
\end{tabular}

\section{PARSNIP}

Long Smooth

Per $1 \mathrm{~b}$

Hollow Crown

$\$ 40$

\section{SEED POTATOES}

Maine or Canadian Grown Certified Seed

Prices Subject to Market

Per 165

ib Sack

Irish Cobbler

.\$ 7.50

Green Mountain

7.50

Spaulding Rose

7.50

MAINE or CANADIAN GROWN-Not Certified Seed

Per 165

tb Sack

Irish Cobbler

$\$ 7.00$

Green Mountain

7.00

Early Northern

7.00

Early Rose

7.00

Spaulding Rose

7.00

Gold Coin

7.00

Carman

7.00

Sir Walter Raleigh

7.00

Delaware

Dibble's Russet

7.50

Bliss Triumph

8.00

Write or wire for delivered carload prices. 


\section{MUSTARD}

$\begin{array}{lr} & \\ \text { Giant Southern Curled } \ldots \ldots \ldots \ldots \ldots \ldots \ldots \ldots \ldots \ldots . . & .30 \\ \text { New Chinese } \ldots \ldots \ldots \ldots \ldots \ldots \ldots \ldots \ldots \ldots \ldots \ldots \ldots \ldots & .30\end{array}$

\section{NASTURTIUM}

Tall Mixed

Per ib

$\$ \quad .35$

Dwarf Mixed

\section{OKRA}

Per th

White Velvet

$\$ \quad .30$

Perkins Long Pod

.30

\section{ONION}

We believe our Onion Seed is best obtainable.

Per to

Extra Early Red Flat

$\$ 1.25$

Wethersfield Large Red

1.25

Southport Red Globe

1.25

Southport Yellow Globe

1.25

Southport White Globe

2.00

Yellow Danvers Flat

1.25

Yellow Globe Danvers

1.25

Yellow Strasburg

1.25

White Portugal

1.75

Prizetaker

1.50

New Queen

1.75

Australian Brown

1.25

Early Yellow Cracker

1.75

$\bar{z} .50$

2.60

1.35

2.50

2.00

Yellow Bermuda

We are headquarters for Pedigreed Onion Seed and Onion Sets, and can furnish any quantity desired. Onion sets are nice and prices very moderate. Our sets are far superior in quality to most of the sets offered. Prices subject to change. Containers at cost.

\section{ONION SETS}

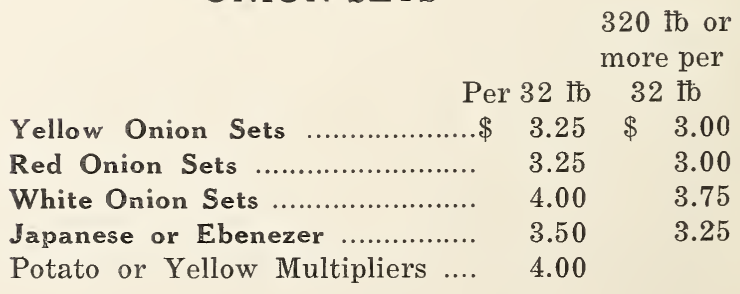




\section{PUMPKIN}

arge Cheese

Small Sugar ....................................... $\quad .50$

Connecticut Field ................................... .35

King of the Mammoth .......................... $\quad .80$

Green Striped Cushaw .......................... $\quad .60$

Yellow or Golden Cushaw ......................... $\quad .60$

White Cushaw ....................................... $\quad .60$

Quaker Pie ............................................. $\quad .60$

Japanese Pie ............................................ $\quad .70$

Tennessee Sweet Potato ............................. $\quad .60$

Big Tom ............................................. $\quad .40$

\section{RADISH}

Vick's Scarlet Globe Selected .................... \$ .35

Scarlet Globe White Tipped ...................... .35

Crimson Giant ..........................................

White Summer Turnip ............................. .35

Early Deep Scarlet ..................................... $\quad .35$

Scarlet Turnip White Tipped ..................... $\quad .35$

French Breakfast .......................................

Olive Shaped Scarlet ............................... .35

Yellow Summer Turnip .......................... . 35

Early Long Scarlet Short Top ................. . .35

Brightest Long Scarlet .......................... $\quad .35$

Philadelphia White Box ......................... .35

White Strasburg ........................................... .35

Long Black Spanish ................................. $\quad .35$

Round Black Spanish ................................ $\quad .35$

China Rose ........................................... $\quad .35$

Half Long Black Spanish ......................... .35

Giant White Stuttgart .............................. $\quad .35$

California Mammoth ................................. 35

White Icicle ........................................... . 35

White Vienna (Lady Finger) .................. $\quad .35$

White Chinese Winter or Celestial ............. $\quad .40$

\section{SPINACH}

Savoy Long Standing .............................. $\quad .18$

Savoy Rust Resistant ................................ .18

Round Thick Leaf ................................... .15

Savoy or Bloomsdale .................. ... . . 18

Viroflay ................................................... .15

Long Standing ...................................... .15

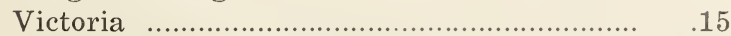

All Seasons or Triumph ......................... 16

New Gaudry Round Seed ......................... 16

King of Denmark ..................................... 18

New Zealand ........................................... .40

Nobel Giant Thick Leaf ............................. $\quad .18$

Juliana ......................................................... . . 


\section{SALSIFY}

Mammoth Sandwich Island

\section{SUNFLOWER}

Mammoth Russian

Per to

$\$ \quad .12$

\section{SORREL}

Per to

Broad Leaved French

$\$ 1.20$

\section{SQUASH}

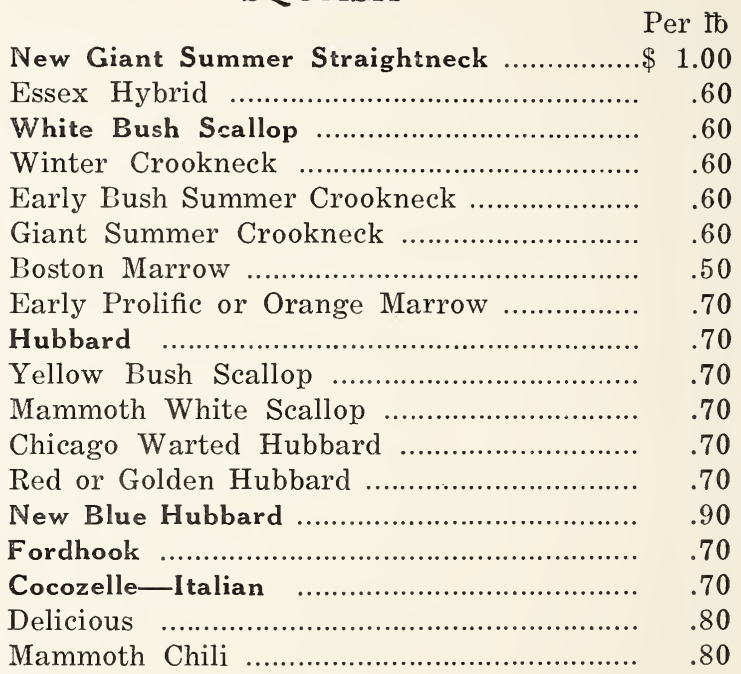

\section{TURNIP}

\section{(White-Fleshed Sorts)}

100 ib or more 5 cents a pound less

\begin{tabular}{|c|c|c|}
\hline & & \\
\hline Purple & e Top Strap Leaved & ...... \\
\hline urple & e Top White Globe & \\
\hline arly & Purple Top Milan & \\
\hline xtra & Early White Milan & \\
\hline hite & Egg & $\ldots \ldots \ldots \ldots \ldots \ldots \ldots \ldots \ldots$ \\
\hline hite & Flat Strap Leave & …................... \\
\hline urple & Top Strap Leaved & \\
\hline
\end{tabular}

Special Select Stock Seed Purple Top White

Globe

Cow Horn or Long White

Pomeranian White Globe

\section{Yellow-Flesh Sorts}

Large Amber Globe 


\section{SWEDES AND RUTABAGA TURNIP}

Per tib

Golden Neckless Rutabaga

$\$ \quad .32$

American Purple Top Rutabaga .................. $\quad .30$

Long Island Purple Top Rutabaga ............. $\quad .30$

White Rock or French ................................ $\quad .35$

Breadstone ................................................ .35

Macomber ................................................ $\quad .50$

\section{TOMATO}

Early Avon

Per itb

Burbank

$\$ 3.00$

Norton's Rust Resistant .......................... 3.00

Fordhook First ...................................... 1.50

Early Acme ........................................... 2.00

Earlibell ................................................... 2.00

Spark's Earliana (true) .......................... 1.50

Dwarf Champion ................................... $\quad 3.00$

Perfection ............................................. 1.25

Florida Special ....................................... 2.00

Cooper's Special ..................................... 2.00

Beauty ............................................... 1.75

Stone ................................................... 1.25

Golden Queen ....................................... 2.50

Ponderosa ............................................ 4.00

John Baer ............................................ 1.50

Chalk's Early Jewel .............................. 1.50

Matchless ................................................ 1.25

Red Rock .............................................. 1.25

Greater Baltimore ................................. 1.50

June Pink ............................................. 3.00

Livingston's Globe .................................. 3.50

Bonnie Best ............................................ 1.50

Marglobe (original stock) ........................ 3.50

Yellow Pear, per oz. ............................... .40

Yellow Plum, per oz. .............................. $\quad .40$

Yellow Cherry, per oz. ............................ $\quad .40$

Red Pear, per oz. .................................... $\quad .40$

Red Plum, per oz. ................................... $\quad .40$

Red Cherry, per oz. ................................. .40

SWEET HERBS

\begin{tabular}{|c|c|c|}
\hline & Per oz. & Per \\
\hline nise & .20 & 15 \\
\hline alm ........ & .30 & \\
\hline Borage $\quad . . .$. & .20 & \\
\hline way & .10 & \\
\hline der & .10 & \\
\hline Dill & .10 & \\
\hline ....... & .30 & \\
\hline der ..... & .40 & \\
\hline Sage, Broad Leaf English ........ & .40 & \\
\hline weet Basil & .30 & \\
\hline weet Fer & .10 & \\
\hline$t$ & .30 & \\
\hline ............. & .40 & \\
\hline $\mathrm{Br}$ & .60 & \\
\hline -Florence or Italia & .30 & \\
\hline
\end{tabular}




\section{S. D. WOODRUFF \& SONS}

\section{FLOWER SEEDS}

\section{Packets 5 cents each}

Per oz. Per ib

Acroclinium, Roseum (double mixed) $\$ .20$

2.25

Roseum (single mixed) .............. .20

Ageratum Mexicanum Blue ........... .20

2.00

Dwarf Blue ............................... . .20

2.50

Dwarf Mixed ............................... .25

2.50

3.00

Agrostemma Coeli Rosa (Rose of

Heaven) ....................................... .15

Coronaria Mixed ......................... $\quad .20$

1.75

1.90

Alyssum Sweet ................................ .15 1.50

Little Gem ............................... $\quad .30 \quad 3.00$

Amaranthus Caudatus (Love Lies

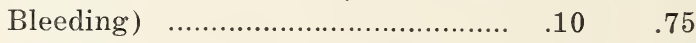

Cruentus .................................... $10 \quad .60$

Tricolor (Joseph's Coat) …......... $.25 \quad 3.00$

Mixed ....................................... $\quad .10 \quad .60$

Antirrhinum Majus Tall, Fine Mixed

Snapdragon ................................... .25

Majus Nanum semi dwarf, mixed .25

3.00

3.00

Tom Thumb Choice Mixed ........... $.50 \quad 6.00$

Aquilegia Single Mixed (Columbine) $\quad .35 \quad 4.60$

Double Mixed ............................... $.40 \quad 5.00$

Arctotis Grandis (African Lilac Daisy ) ...................................... .25

Asperula Azurea Setosa Blue ......... .15

2.75

Aster Giant Branching White ........... $\quad .50 \quad 6.00$

Pink ............................................ $\quad .50 \quad 6.00$

Crimson ....................................... $.50 \quad 6.00$

Dark Blue ................................... $\quad .50 \quad 6.00$

Lavender .................................... $.50 \quad 6.00$

Rose .......................................... .50 6.00

Mixed ............................................... $\quad .40 \quad 5.50$

Queen of the Market Mixed .......... $\quad .40 \quad 5.00$

Improved Victoria Mixed ............. $\quad .75 \quad 9.00$

Improved Crego ............................ $.55 \quad 6.50$

$\begin{array}{llll}\text { Trauffauts Peony Perfection Mixed } & .60 & 7.00\end{array}$

American Beauty Mixed ............. $\quad .75 \quad 9.50$

Chinese Single Mixed .................. .40 5.00

Balsam Tall Double Mixed ............. .15 1.30

Dwarf Double Mixed ................... .25 $\quad 2.75$

Balsam Camelia Flowered Choice Mixed ........................................... .25

Bellis Mixed (Double Daisy) ........... $.80 \quad 10.50$

Brachycome Iberidifolio (Swan River Daisy) Blue .................................. . .35 4.50

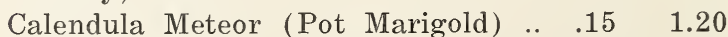

Double Sulphur Yellow ............... $.15 \quad 1.20$

Orange King .................................. .15 $\quad 1.10$

Prince of Orange ....................... $.15 \quad 1.10$

Mixed ........................................ $\quad .10 \quad 1.00$

Calliopsis Mixed, Annual Sorts ....... $.15 \quad 1.25$

Drummondi (Golden Wave) ....... $.15 \quad 1.50$

Campanula Medium White ............. $\quad .25 \quad 2.75$ 


\section{FLOWER SEEDS-Continued}

\begin{tabular}{|c|c|c|}
\hline & & \\
\hline Ledium Blue & .25 & $\$$ \\
\hline edium Rose & .25 & \\
\hline Double Mixed & .60 & \\
\hline anary Bird Flower & .15 & \\
\hline inna Crozys (New Hybrid Mixed) & .30 & \\
\hline indytuft White Rocket .................. & .10 & \\
\hline Giant Empress ................................ & .30 & \\
\hline Lilac & .25 & \\
\hline Rose & .30 & \\
\hline All Sorts Mixed & .10 & \\
\hline arnation Marguerite Double Mixed & .75 & \\
\hline warf Vienna Mixed ........................... & .70 & \\
\hline - Oil Bean, Mixed .. & .10 & \\
\hline ardinal Climber. & .60 & \\
\hline $\begin{array}{c}\text { elosia, Cristata Tall Mixed (Cox- } \\
\text { comb) }\end{array}$ & .20 & \\
\hline sa Feathered, Tall Mixed .... & .15 & \\
\hline ii Crimson (Chinese Wool & & \\
\hline 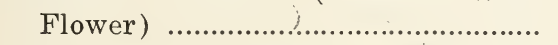 & .75 & \\
\hline cea Candidissima (Dusty Mil- & & \\
\hline 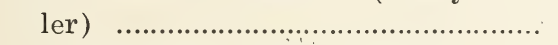 & 1.25 & \\
\hline nus Blue (Corn Flower) ........ & .15 & \\
\hline Nhite & .15 & \\
\hline S Rose & .15 & \\
\hline$\ldots \ldots \ldots \ldots \ldots \ldots$ & .15 & \\
\hline .................. & .20 & \\
\hline Sultan) .. & .30 & \\
\hline m Double & & \\
\hline & .1 & \\
\hline (n).................. & .15 & \\
\hline idum (Shasta & & \\
\hline & .00 & \\
\hline e Mixed .. & .20 & \\
\hline$d \ldots \ldots \ldots \ldots \ldots \ldots \ldots$ & .2 & \\
\hline .......................... & .2 & \\
\hline Convolvulus Major Tall Mixed & & \\
\hline & .10 & \\
\hline lixed ....................... & .1 & \\
\hline Ay Lenox White ............... & .15 & 5 \\
\hline Lady Lenox Pink .................... & .15 & \\
\hline Lady Lenox Crimson & .1 & \\
\hline $\mathrm{t} N$ & .1 & \\
\hline Early Flowering Man & .20 & \\
\hline Early Flowering Mammoth Pink .. & .20 & 1 \\
\hline Early Flowering Mammoth Crim- & & \\
\hline ........ & .2 & \\
\hline Early Flowering Mammoth Mixed & .15 & \\
\hline Tivo & 10 & \\
\hline & & \\
\hline $\operatorname{lpl}$ & .50 & \\
\hline
\end{tabular}




\section{$16 \quad$ S. D. WOODRUFF \& SONS}

\section{FLOWER SEEDS-Continued}

Dianthus Chinese Double Mixed ..... $.25 \quad 2.75$

Chinese Single Mixed ................... $.20 \quad 2.50$

Heddewigii Double Mixed ........... $.35 \quad 4.50$

Heddewigii Single Mixed ............. .25 $\quad .250$

Plumarius Double Mixed ............. 1.00

Plumarius Single Mixed ............... $.25 \quad 2.80$

Laciniatus Double Mixed ............. $.50 \quad 6.00$

Laciniatus Single Mixed .............. . .30 3.25

Digitalis (Foxglove) Purpurea Mixed $\$ .25 \$ 2.75$

Dolichos Mixed (Hyacinth Bean) $\quad .20 \quad .65$

Echinocystis Lobata (Wild Cucumber)

Escholtzia California Yellow (California Poppy) ............................. .15

All Sorts Mixed ........................... .15

1.25

1.10

Euphorbia Variegata (Snow on the Mt.)

Gaillardia Grandiflora Single Mixed.. $.25 \quad 2.50$

Lorenziana Double Mixed ........... . .25 $\quad .75$

Godetia Tall Mixed ....................... .20 2.25

Dwarf Mixed ................................ $\quad .20 \quad 2.50$

Gomphrena Globesa (Globe Amaranth) Mixed .................................. .15

1.50

Gourds (See Page 7)

Grasses Ornamental, Mixed ............. . .20

Gypsophila Elegans White ............... .10

Elegans Mixed ........................... .15

1.90

.75

1.25

Helichrysum Fine Mixed ................ . .30 3.75

Monstresum Fine Double Mixed .. $.30 \quad 3.75$

Heliotrope Choice Mixed ................. .60 $\quad 7.50$

Heterophy (Mexican Fire Plant) ..... $\quad .30 \quad 3.75$

Hibiscus Africanus ......................... .40 5.00

Humulus Japonicus (Japanese Hop).. $\quad .25 \quad 2.75$

Hollyhock Everblooming Annual

Single Mixed ............................. . .35 3.75

Everblooming, Annual Double

Mixed ........................................... .40 4.50

Iceplant ........................................... $\quad .20 \quad 2.00$

Ipomea Noctiflora (Moonflower) ....... $\quad .25 \quad 2.75$

Stetosa (Brazilian Morning Glory) $\quad .20 \quad 2.40$

Imperialis (Japanese Morning

Glory) ........................................ $\quad .10 \quad .65$

Quamoclit (Cypress Vine) ............ $.15 \quad 1.75$

Kochia (Summer Cypress) ............... $.10 \quad 1.00$

Larkspur Tall Rocket Mixed Double $.25 \quad 3.50$

Double Dwarf Rocket Mixed ............ . .25 3.50

Lavendar Lace Flower ..................... $\quad .75 \quad 9.00$

Linum Grandifforum Rubrium ......... $\quad .15 \quad 1.75$

Lobelia Erinus Crystal Palace True $1.50 \quad 20.00$

Erinus Gracilis Blue .................. .40 4.50

Erinus Fine Mixed ...................... $.50 \quad 6.50$

Lupinus Tall Annual Mixed ........... $.15 \quad 1.50$

Dwarf Annual Mixed ................... $\quad .25 \quad 3.00$

Marigold African Orange Queen .... $.35 \quad 4.50$ 


\section{FLOWER SEEDS-Continued}

Per oz. Per to

African Lemon Queen ................ .35 4.50

African Eldorado Queen ............. $.30 \quad 4.00$

African Dwarf Mixed ................ . .30 4.00

African Tall Mixed .................... $.30 \quad 4.00$

French Tall Mixed ........................ $.25 \quad 3.00$

French Dwarf Mixed .................. .25 $\quad 3.00$

Mathiola Bicornis ......................... \$ $.15 \$ 1.50$

Matricaria Capensis Fl. Pl. (Feverfew) .......................................... $.25 \quad 3.00$

Marvel of Peru Mixed (four-o'clock) $\quad .10 \quad 1.00$

Mignonette Sweet ........................... .10 1.00

Allens Defiance ........................... .25 3.00

Morning Glory, See Convolvulus and Ipomea.

Myosotis Alpestris Blue (Forget-Me-

Not) ........................................... .40 4.50

Alpestris Mixed ........................... .40 4.50

Nasturtium Dwarf Mixed ................ . . $10 \quad .35$

Tall Mixed ........................................ . . 10 .35

Nemophila, Best Mixed .................. $\quad .10 \quad 1.00$

Nicotiana Affinis ............................ .20 2.10

Affinis Hybrid .............................. .25 $\quad .250$

Pansy Giant Trimardeau Mixed ....... $.90 \quad 11.50$

French Mixed ............................ $.80 \quad 10.00$

English Mixed ........................... $\quad .80 \quad 10.00$

Choice Mixed .............................. $\quad .60 \quad 7.00$

Petunia Choice Mixed ..................... . .60 7.50

Fine Mixed ................................. .40 4.40

Phlox Drummondi Fine Mixed .......... $\quad .40 \quad 5.00$

Drummondi Extra Choice Mixed .. $\quad .50 \quad 6.00$

Poppy Mudicaule (Iceland) ............. $\quad .70 \quad 9.00$

Orientale, Scarlet .......................... $\quad .80 \quad 10.00$

Carnation Flowered Best Mixed .. $\quad .10 \quad .100$

Shirley Single Mixed ................... $.10 \quad 1.00$

All Sorts Mixed Single ................ $.15 \quad 1.25$

Double Mixed, All Sorts ............ . . 10 $\quad .75$

Portulaca Single Mixed ................. . .35 $\quad 4.00$

Double Mixed ........................... .25 2.75

Pyrethrum Aurem (Golden Feather ) $\quad .25 \quad 2.75$

Salpiglossis Fine Mixed .................. .30 4.00

Salvia Splendens (Scarlet Sage) $\ldots . .1 .25 \quad 18.00$

Bonfire ...................................... $1.75 \quad 25.00$

Scabiosa Tall Mixed ........................ .25 3.00

Snapdragon, See Antirrhinum

Stocks 10 Weeks Double Mixed ........ $\quad .90 \quad 13.00$

Giant Perfection Choice (Cut and

Come Again) Mixed .................... $1.30 \quad 18.00$

Sunflower Globosus Mixed ............... $\quad .15 \quad 1.40$

Double California Yellow ........... $15 \quad 1.40$

Annus Tall Double Mixed ........... $.25 \quad 3.00$ 


\section{FLOWER SEEDS—Continued}

\section{SWEET PEAS \\ Spencer Varieries}

Appleblossom, Rose with Primrose

Countess Spencer, Pink, Fiery Cross, Orange Scarlet, Florence Nightingale, Lavender.

Helen Lewis, Orange Salmon.

King Edward VII, Red.

King Manuel, Chocolate Maroon.

King White, Pure White.

Royal Purple, Purple.

Wedgewood, Blue.

Per $1 / 4$ ib Per 1b

All of the above varieties ...........\$ $.35 \$ 1.20$

Superb Spencer, mixed, 100 tb lot at $\quad .75 \quad .90$

Grandiflora Choice, mixed, 100 ib lot

at ............................................... . .35

Trial Ground, mixture, 100 fb lots at $\quad .35 \quad .40$

Per oz. Per ib

Sweet William, single mixed

$\$ .20 \$ 2.25$

Sweet William, double mixed .......... $.40 \quad 5.00$

Verbena Hybrida, fine bixed ............. $.35 \quad 4.50$

Verbena, Mammoth mixed ............... .35 $\quad .50$

Vinca Rosea, mixed ........................ $.50 \quad 6.00$

Wallflower, single mixed ................ $\quad .20 \quad 2.00$

Wallflower, double mixed ............... $1.50 \quad 22.00$

Wallflower, Annual Blood Red ........ ..35

Xeranthemum, Everlasting double mixed ......................................... .35 3.50

Zinnia, Elegans, Tall double mixed.. $.20 \quad 1.75$

Zinnia, Dwarf, double mixed ......... $.20 \quad 1.75$

Zinnia, Giant, double mixed ........... $.35 \quad 3.50$

\section{GLADIOLI}

Doz. Per 100

Marshall Foch xx Pink

$\$ 1.20 \$ 4.00$

Panama (Salmon)

1.20

4.00

Gladioli Bulbs, mixed 1st size

1.00

3.00

Gladioli Bulbs, mixed 2nd size

2.50

Rhubarb Roots

$2.00 \quad 10.00$

Horse Radish Roots

$1.00 \quad 8.00$

SEED OATS

Per bu. 32 1b

Lincoln

.\$ 1.35

White Cluster

1.35

Silver Mine

1.35

Big Four

White Russian

Swedish Select

White Tartar

Conqueror, new 


\section{SEED GRAINS}

Barley Hulless

Per bu.

Barley, 6 Row, 48 it

$\$ 2.50$

Spring Rye, 56 ib

2.25

Winter Rye, 56 ib

2.75

2.00

Spring Wheat, 60 tb

3.50

Winter Wheat, white, $60 \mathrm{lb}$

3.50

Winter Wheat, white, $60 \mathrm{lb}$

3.50

Winter Wheat, red, 60 ib

3.50

Buckwheat, Japanese, 48 ib

3.50

\section{FIELD AND COW PEAS}

Medium Green Cow Peas

$\$ 3.75$

Black Cow Peas

3.75

Whippoorwill Peas

3.75

Soy Beans

4.00

White Carada Field Peas

4.00

\section{VETCH}

Spring Vetch or Tares

Per to

Winter Sand or Hairy

$\$ \quad .10$

.20

\section{MILLET}

German or Golden

Hungarian

Japanese XX

Market Gardener's Catologue mailed on on application.

Don't fail to include in your order, some of Woodruff's Mixed Lawn Grass Seed, per measured bushel, $\$ 4.00$.

\section{GRASS SEED}

We offer the following Timothy and Clover Seeds, as follows:

Fancy Timothy, $993 \frac{1}{4} \%$ pure $\$ .081 / 2$

Choice Timothy, $991 / 2 \%$ pure

Fancy Medium Red Clover ....

Fancy Mam. Red Clover ............................. $\quad .30$

White Clover ............................................... $\quad .40$

Alfalfa Clover (northwestern) .................. $\quad .30$

Alsike Clover .......................................... $\quad .32$

Crimson Clover ......................................... 14

Grimm Alfalfa ..................................... . .45 


\section{Woodruff's High-Grade Grass Seeds}

Fancy Red Top XX

Per $1 \mathrm{~b}$

Unhulled Red Top

$\$ .22$

Kentucky Blue Grass

.15

Orchard Grass (14 ib per bu.)

.30

Crested Dog Tail

.22

Italian Rye Grass

English Rye Grass

Meadow Fescue

Sheep Fescue

Creeping Bent, German ............................ 1.00

Hard Fescue ........................................... $\quad .30$

Meadow Foxtail ....................................... $\quad .30$

Tall Meadow Grass .................................... $\quad .30$

Sudan Grass ............................................ $\quad .12$

Dwarf Essex Rape ................................ $\quad .10$

\section{Woodruff's Standard Lawn Grass Seed}

$20 \mathrm{lb}$. per bu.

One Quart Cartons Velvet Green, Central

Lawn or Shady Nook Lawn Seed in cases 2

dozen per case

GRASS MIXTURE FOR GOLF LINKS, TENNIS COURTS, ETC.

Fair Green Mixture

For the Grounds in general use Woodruff's

Special Mixture, bushel

\section{Putting Green Mixture}

24 ib to bu.

Peck

Bushel

Tennis Court Mixture

Tennis courts need Grasses of a close, compact growth; 24 ib to bu.

Peck

Bushel

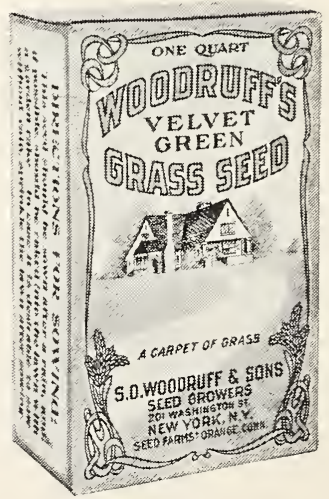




\section{Shady Nook Mixture}

4 bushels to acre, 24 ib to bu.

Peck

Bushel

\section{Terrace Sod Mixture}

$24 \mathrm{lb}$ to bu.

Peck

Bushel

Woodruff's Lawn Dressing Fertilizer $\$ 43.00$ per ton in $100 \mathrm{fb}$ bags

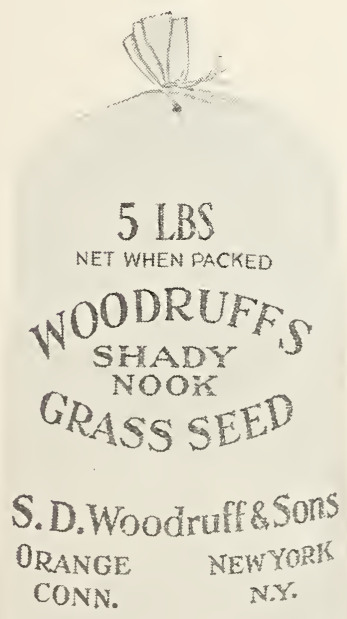

"SHADY NOOK" GRASS SEED

\section{It Grows}

In The Shade 


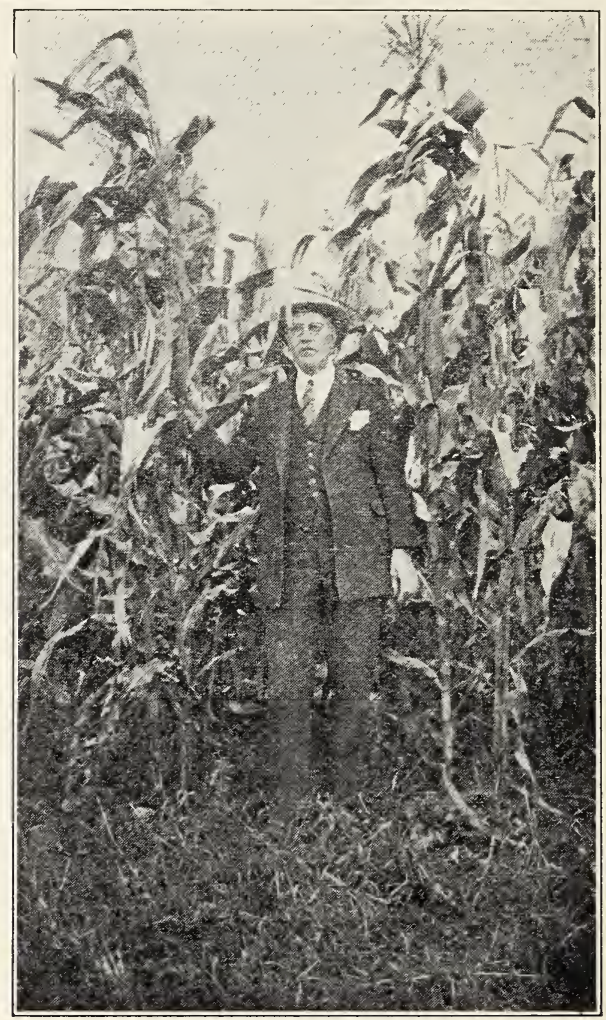

\section{WOODRUFF'S BEAUTY SILO CORN}

The most profitable corn that goes into the silo. Grows 10 to 14 feet. Early and matures its rich golden ears even in Northern New England. From a few bushels of seed first put out by us in 1923, our dairymen of the East now require from us many thousands of bushels annually. Per bu. $\$ 3.00$.

S. D. Woodruff \& Sons,

Dec. 14

Orange, Conn.,

Gentlemen :

The above is a photograph of WOODRUFF'S SELECTED BEAUTY ENSILAGE CORN on the Holy Cross College Farm of Worcester, Mass. It was exactly 95 days from planting to cutting. The germination was practically $100 \%$, grew on an average of $12 \mathrm{ft}$. high and $75 \%$ of the stalks had two ears which measured on an average of 18 inches long. The ears were filled and fully matured for silo.

J. B. GARLAND \& SON, per William L. Little, 15 Grafton St., Worcester, Mass.

(Write for Dealers Special Discount on Pedigreed Seed Corn in quantity.) 


\section{SEEDS IN PACKETS}

We offer our standard sized packets of both GARDEN and FLOWER SEEDS, excepting the heavier items which we supply in bulk, namely, Peas, Beans and Sweet Corn, as follows:

\section{All following packets retail at $10 \mathrm{c}$ each}

Net trade price per 100 packets

Or in lots of 500 and over, either of one variety or assorted, price is per 100 packets .. 4.00

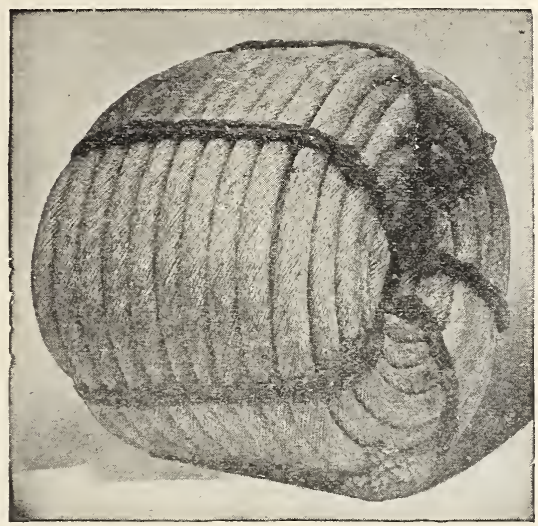

TYING TWINE

2, 3, or 4-ply-Specify which.

This twine is in the form of a loose rope (100 strings in the rope) and wound on a reel the same as barbed wire. In this manner it never becomes snarled and enables one to cut off a hundred strings any desired length instantly.

Price per reel of $50 \mathrm{ib}, 20 \mathrm{c}$ per $1 \mathrm{~b}$.

\section{HOT BED MATS}

Burlap both sides

$40 \times 76$ in.

$76 \times 76$ in.

$76 \times 84$ in.
Each 1/2 Doz. Doz.

$\$ 2.00 \quad \$ 10.50 \quad \$ 18.00$

$3.25 \quad 18.00 \quad 33.00$

$3.50 \quad 19.50 \quad 35.00$

Prices are F. O. B. Connecticut, net cash with order. 


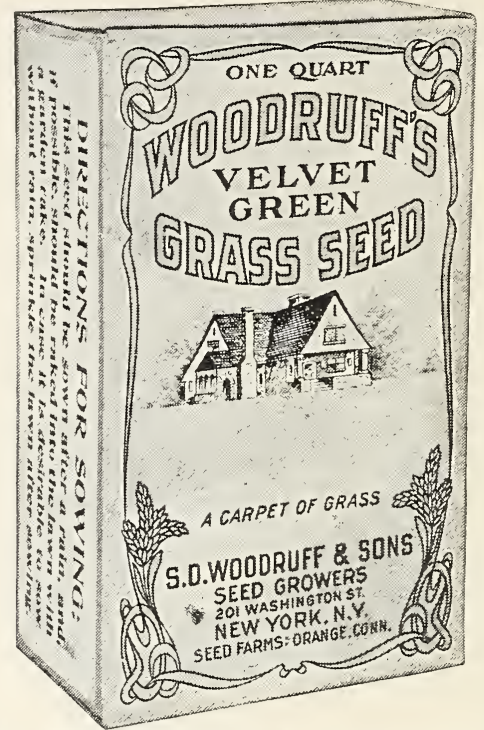

\section{WOODRUFF'S LAWN GRASS MIXTURES} The Most Profitable Quick Turning Item a Dealer Can Stock

\section{VELVET GREEN LAWN SEED}

\section{"A CARPET OF GRASS"}

100 lb. bag .....................\$20.00 per 100 lbs. $500 \mathrm{lb}$. or more ................. 18.00 per 100 lbs. These mixtures also packed in $5 \mathrm{lb}$. White Cotton Bags. These can be printed with your own name on the Bags if you want them in lots of 100 Packages or more. A very attractive Package Price of Five Pound Bags-Two cents Per 1b. more than the $100 \mathrm{lb}$. Price.

WOODRUFF'S CENTRAL LAWN SEED 100 lb. bag ......................\$18.00 per 100 lbs. 500 lbs. or more 16.00 per $100 \mathrm{lbs}$.

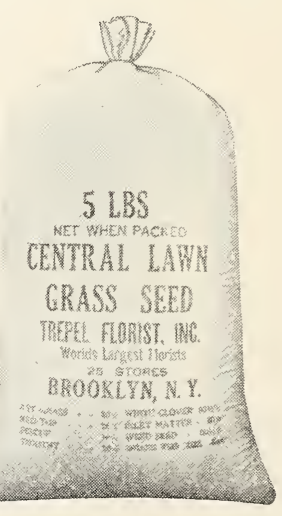

SHADY NOOK LAWN SEED

100 lb. bag $\$ 22.00$ per 100 lbs. $500 \mathrm{lbs}$. or more 20.00 per 100 lbs. 


\section{SHEEP MANURE}

\section{For Lawn Dressing}

ANALYSIS

Ammonia $.2 .50 \%$

Soluble Potash $2.00 \%$

Phosphoric Acid $1.00 \%$

Odorless, easy to apply, quick acting.

3 ib package

5 tb package

100 ib package

Ton in 100 ib bags

\section{BONE MEAL}

3 tb package ....................................... .20

5 tb package ........................................

50 it bag ............................................ 3.00

Ton in $100 \mathrm{tb}$ bags ............................ 50.00

\section{WOODRUFF'S HOME MIXED FERTILIZERS}

We offer the following formula:

Ammonia $4.00 \%$

Available Phos. Acid $8.00 \%$

Potash $.6 .00 \%$

Spot cash price, $\$ 43.00$ per ton,

F. O. B. Orange, Conn.

Per 100 tb $\$ 3.50$

Nitrate of Soda in original bags, about 225 ib each at $\$ 3.75$ per $100 \mathrm{lb}$.

Ground Castor Pomace, guaranteed $5 \frac{1}{2} \%$ Ammonia, at $\$ 2.50$ per 100 ib.

Acid Phosphate guaranteed $16 \%$ available Phosphoric Acid, at $\$ 2.25$ per 100 ib.

In addition to the above, we offer the following: Commercial Fertilizers, F. O. B., New York City:

\section{COMPLETE MANURE}

$2 \%$ Ammonia

8\% Phosphoric Acid

$3 \%$ Potash at $\$ 38.00$ per Ton

\section{GARDEN AND TRUCK FERTILIZER}

$2 \%$ Ammonia

$8 \%$ Phosphoric Acid

$4 \%$ Potash at $\$ 42.00$ per Ton

\section{GOLDEN HARVEST SPECIAL}

4\% Ammonia

8\% Phosphoric Acid

$4 \%$ Potash at $\$ 46.00$ per Ton All in 100 pound bags. Terms cash. 


\section{WOODRUFF'S HOME MIXED SPECIAL FERTILIZER}

Ammonia

$.5 .00 \%$

Available Phos. Acid $.8 .00 \%$

Potash $.7 .00 \%$

Cash price $\$ 45.00$ per ton, F. O. B. Orange, Conn.

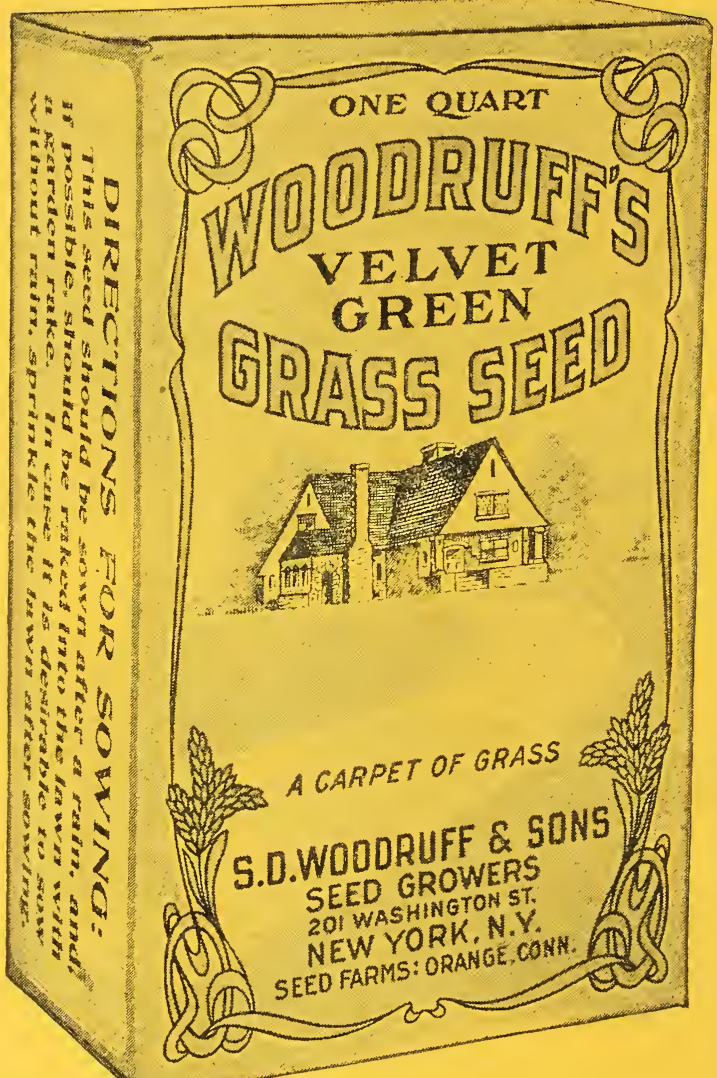

VELVET GREEN LAWN SEED

"A Carpet of Grass"

(Packed in Cartons-24 to a Case)

Per Case $\$ 3.25$ Per Case

Five Cases or More $\$ 3.15$

S. D, WOODRUFF \& SONS

Orange, Conn.
201 Washington St., New York, N. Y. 